\title{
Age-related decrease in motor cortical inhibition during standing under different sensory conditions
}

\author{
Selma Papegaaij ${ }^{1}$, Wolfgang Taube ${ }^{2}$, Margot Hogenhout ${ }^{1}$, Stéphane Baudry ${ }^{3}$ and Tibor Hortobágyi ${ }^{1,4}$ \\ ${ }^{1}$ Center for Human Movement Sciences, University of Groningen, University Medical Center Groningen, Groningen, Netherlands \\ ${ }^{2}$ Movement and Sports Science, Department of Medicine, University of Fribourg, Fribourg, Switzerland \\ ${ }^{3}$ Laboratory of Applied Biology, Faculty for Motor Sciences, Université Libre de Bruxelles, Brussels, Belgium \\ ${ }^{4}$ Faculty of Health and Life Sciences, Northumbria University, Newcastle Upon Tyne, UK
}

\section{Edited by: \\ Jesús Avila de Grado, Centro de Biología Molecular Severo Ochoa - \\ Consejo Superior de Investigaciones Científicas-Universidad Autónoma de Madrid, Spain}

\section{Reviewed by:}

Rongqiao He, Institute of Biophysics Chinese Academy of Sciences, China Patrick Ragert, Max Planck Institute for Human Cognitive and Brain Sciences Leipzig, Germany

\section{*Correspondence:}

Selma Papegaaij, Center for Human Movement Sciences, University of Groningen, University Medical Center Groningen, Antonius Deusinglaan 1, 9713AV Groningen, Netherlands e-mail: s.papegaaij@umcg.nI

\begin{abstract}
Background: Although recent studies point to the involvement of the primary motor cortex in postural control, it is unknown if age-related deterioration of postural control is associated with changes in motor cortical circuits. We examined the interaction between age and sensory condition in the excitability of intracortical motor pathways as indexed by shortinterval intracortical inhibition (SICl) and intracortical facilitation (ICF) during standing.
\end{abstract}

Methods: We used magnetic brain stimulation to evoke SICl and ICF in 11 young (range 2125 years) and 12 healthy old adults (range 60-74 years) while they stood on a rigid platform or foam, with the eyes open or closed.

Results: There was an overall age-related $43 \%$ reduction in $\mathrm{SICI}(p=0.001)$. SICI lessened when standing on foam in old $(31 \%)$ but not in young $(1 \%)$ adults (condition $\times$ group interaction, $p=0.049$ ). This reduction was associated with increases in center of pressure velocity $(r=-0.648, p=0.043)$. Age $(p=0.527)$ and sensory conditions $(p=0.325)$ did not affect ICF.

Conclusion: Motor cortical circuits controlling leg muscles are modulated differently in healthy old vs. young adults during upright posture. Future experiments will clarify whether this difference mediates impaired postural control or serves as a compensatory mechanism to counteract postural instability.

Keywords: balance, aging, brain aging, transcranial magnetic stimulation, short-interval intracortical inhibition

\section{INTRODUCTION}

Accumulating evidence points to the involvement of the primary motor cortex (M1) in postural control (Beloozerova et al., 2003; Taube et al., 2006, 2008; Tokuno et al., 2009). Several studies also suggest that training of balance skills is associated with M1 plasticity (Beck et al., 2007; Schubert et al., 2008) and that this plasticity correlates with improvements in postural control (Taube et al., 2007; Taubert et al., 2010). It is well known that postural control of upright stance progressively declines with age. Many factors have been identified to contribute to the age-related deterioration of postural control, including reduced muscle strength (Boelens et al., 2013), impaired sensory abilities (Proske and Gandevia, 2012), slowed nerve conduction velocity (Nardone et al., 1995), and altered spinal reflexes (Koceja et al., 1995; Baudry and Duchateau, 2012). In addition, the excitability of the corticospinal pathway, including the direct projections (monosynaptic) from cortical neurons to spinal motor neurons, is greater in old compared with young adults during upright standing (Baudry et al., 2014a,b). It is unknown if, in addition to age-related changes in the corticospinal pathway, aging also influences intracortical circuits during upright standing.

During manual motor tasks, neuroimaging studies report greater activation in M1, premotor, and prefrontal areas in old compared with young adults (Calautti et al., 2001; Mattay et al.,
2002; Ward and Frackowiak, 2003). Similarly, studies using transcranial magnetic stimulation (TMS) revealed disinhibition in several M1 inhibitory circuits in old compared with young adults during manual motor tasks, as reflected by decreases in interhemispheric inhibition, silent period, cortical reciprocal inhibition, and short-interval intracortical inhibition (Oliviero et al., 2006; Hortobagyi et al., 2006; Talelli et al., 2008; Marneweck et al., 2011; Fujiyama et al., 2012; Heise et al., 2013). However, several other studies report no changes (Oliviero et al., 2006; Smith et al., 2011) or even increased inhibition with age (Kossev et al., 2002; McGinley et al., 2010). These inconsistencies in literature are probably related to differences in task difficulty, age range and the type of muscle examined. Age-related decreases in intracortical inhibition have been associated with worse motor performance measured under a variety of experimental conditions (Marneweck et al., 2011; Fujiyama et al., 2012; Heise et al., 2013). Thus, there is ample evidence that aging modifies intracortical processing and that such modifications can affect motor control (for a review, see Papegaaij et al., 2014). Hence, it seems reasonable to expect that age-related changes in intracortical circuits are - at least in part responsible for the deterioration in postural control.

Therefore, the purpose of the present study was to examine the interaction between age and sensory condition in M1 excitability as indexed by short-interval intracortical inhibition (SICI) and 
intracortical facilitation (ICF) during standing. We manipulated sensory condition by altering proprioceptive feedback (standing on a rigid platform vs. foam) and visual feedback (eyes open vs. eyes closed).

Based on work showing modulations in inhibitory spinal circuits with sensory conditions and postural task difficulty (Koceja et al., 1995; Katz et al., 1988; Baudry and Duchateau, 2012), we hypothesized that behaviorally relevant modulations would also occur in M1 excitability. Specifically, we expected a reduction of SICI with altered sensory conditions, to increase M1 excitability and consequently the number of motor solutions available. In addition, based on the previously mentioned studies reporting cortical disinhibition with aging (Oliviero et al., 2006; Hortobagyi et al., 2006; Talelli et al., 2008; Marneweck et al., 2011; Fujiyama et al., 2012; Heise et al., 2013), we expected that old adults would show a generally reduced SICI compared to young adults. Based on the findings of Heise et al. (2013), we hypothesized that this age-related disinhibition during normal standing would result in a decreased modulation of SICI between tasks. To validate the putative role of M1 in postural control, we predicted an association between the task-related modulation of M1 excitability and alteration in postural stability. Because of a lack of data concerning ICF modulation during postural tasks, the formulation of a specific hypothesis is premature.

\section{MATERIALS AND METHODS PARTICIPANTS}

Eleven healthy young adults (age $23 \pm 1$ years, range $21-25$ years, 4 men) and fourteen healthy old adults (age $68 \pm 5$ years, range 60-77 years, 11 men) volunteered for the study. In two old men (age 66 and 77 years) the stimulation intensity was above the comfort threshold and we stopped data collection. Two young and two old adults were left-footed (Hebbal and Mysorekar, 2006). None of the participants had a history of or presented with neurological disorders, severe orthopedic disorders, suspicion of pregnancy, non-dental associated metal within the cranium, or took neuroactive drugs or drugs known to affect balance. The mini-mental state examination (MMSE) and short questionnaire to assess health-enhancing physical activity (SQUASH) were used to determine general cognitive function and physical activity in daily life. Subjects also completed the short physical performance battery (SPPB) including standing balance, walking speed and chair stand tests to specifically evaluate lower extremity function (Table 1). Before the experiment, subjects signed an informed consent document approved by the Medical Ethics Committee of the University Medical Center Groningen.

\section{EXPERIMENTAL SETUP}

Surface electromyography (EMG) was recorded (DE-2.1, Delsys, Natick, MA, USA) for the right tibialis anterior (TA) by attaching active electrodes over the muscle belly and the reference electrode on the medial aspect of the tibia. To minimize impedance at the electrode-skin contact, the skin was shaven, abraded with fine-grain sandpaper, and cleaned with alcohol. The EMG signal was amplified 1000 times (model Bagnoli-8, Delsys, Natick, MA, USA), sampled at $5 \mathrm{kHz}$, and bandpass filtered
Table 1 | Subject characteristics.

\begin{tabular}{lll}
\hline & Young adults & Old adults \\
\hline Age (years) & $23 \pm 1$ & $68 \pm 4$ \\
Sex (male; female) & $4 ; 7$ & $11 ; 3$ \\
BMI (kg/m²) & $23 \pm 1.7$ & $24 \pm 3.6$ \\
SPPB score & $12 \pm 0$ & $12 \pm 1$ \\
MMSE score & $30 \pm 0$ & $30 \pm 1$ \\
SQUASH & & \\
Total score & $9718 \pm 1934$ & $10045 \pm 3236$ \\
Light (min/w) & $2337 \pm 580$ & $912 \pm 829$ \\
Moderate (min/w) & $459 \pm 309$ & $353 \pm 284$ \\
Heavy (min/w) & $222 \pm 229$ & $602 \pm 218$
\end{tabular}

Values are mean $\pm S D$, unless indicated differently. BMI: body mass index, SPPB: short physical performance battery (max. score of 12), MMSE: mini mental state examination (max. score of 30), SQUASH: short questionnaire to assess healthenhancing physical activity. Total score is minutes per week $\times$ intensity of the activity. The amount of light, moderate and heavy exercise is expressed in minutes per week.

with a second order Butterworth filter (10-1000 Hz) using data acquisition interface and software (Power 1401 and Spike2, Cambridge Electronics Design, Cambridge, UK). Subjects performed a maximum voluntary contraction (MVC) of the ankle dorsal flexors while seated in a chair with the knee in $45^{\circ}$ flexion and the ankle in neutral position. The EMG activity recorded during this effort was used to express and normalize the background EMG.

During the main part of the experiment, subjects were instructed to maintain an upright bipedal stance on two force plates (Bertec 4060-08, Columbus, OH, USA). With the arms crossed across the chest, subjects looked at a sharply visible "+" sign displayed on a projection screen. Foot position was standardized with the heels $9 \mathrm{~cm}$ apart and a toe-out angle of $30^{\circ}$. The center of pressure $(\mathrm{CoP})$ position signal was sampled at $100 \mathrm{~Hz}$, and filtered using a fourth order low-pass Butterworth filter with a cut off frequency of $10 \mathrm{~Hz}$. It was not necessary to correct the CoP position data for height of the foam $(6 \mathrm{~cm})$ because pilot experiments showed a minimal effect.

An 11-video-camera motion analysis system (Vicon, Oxford, UK) recorded spatial coordinates of reflective markers placed on the right trochanter major and lateral malleolus. The signal was sampled at $100 \mathrm{~Hz}$, filtered with a second order low-pass Butterworth filter (cut off frequency: $5 \mathrm{~Hz}$ ), and used online to determine whether the person was swaying forward or backward.

In a random order, subjects performed four standing conditions with altered sensory states: standing on a rigid surface with eyes open (rigid - EO) and eyes closed (rigid - EC), standing on foam $(47 \mathrm{~cm} \times 38 \mathrm{~cm} \times 6 \mathrm{~cm}$, Bodybow Healthcare BV, Nieuwegein, Netherlands) with eyes open (foam - EO) and eyes closed (foam - EC). Three minute resting periods were given between conditions to prevent fatigue. We also recorded two periods of $10 \mathrm{~s}$ per surface condition (rigid, foam) where participants assumed the standardized position without receiving TMS. 


\section{TMS DATA ACOUISITION}

In the present study, transcranial magnetic stimuli were delivered over the left M1 with a double cone coil connected to a Magstim $200^{2}$ and Bistim ${ }^{2}$ (Magstim, Whitland, UK), except for two old adults for whom a $90 \mathrm{~mm}$ circular coil was used as no clear MEP was evoked with the double-cone coil. We targeted the TA because this muscle generally provides a low motor threshold (MT) and good reliability of MEPs (Cacchio et al., 2009). The optimal location for eliciting MEP's in the TA with the largest amplitude at a given intensity was determined by moving the coil systematically in steps of $0.5 \mathrm{~cm}$ over the M1 area starting at the vertex. In general, the optimal location was found $0.5-1.5 \mathrm{~cm}$ posterior and lateral to the vertex. The location was marked on the skull with a permanent marker to enable the experimenter to hold the coil on a consistent location throughout the experiment. While standing, the MT, defined as the lowest intensity in which the MEP's were larger than $100 \mu \mathrm{V}$ in at least three out of five consecutive trials, was determined (Calautti et al., 2001).

Paired-pulse TMS with an interstimulus interval of $2.5 \mathrm{~ms}$ was used to assess SICI, while an interstimulus interval of $13 \mathrm{~ms}$ was used to assess ICF. The interstimulus intervals were chosen based on the literature (Roshan et al., 2003; Soto et al., 2006) and pilot experiments showing greatest inhibition and facilitation at these intervals. Conditioning and test stimulation intensity were set at 0.8 and 1.2 MT, respectively. In all standing conditions there were 10 test MEP, 10 SICI, and 10 ICF trials. To reduce variability in MEP size induced by sway direction (Tokuno et al., 2009) and to minimize the magnitude of background EMG, TMS was triggered only when the person was swaying forward (negative angular velocity) and with a minimal interval of $5 \mathrm{~s}$ between trials.

\section{DATA ANALYSIS}

Rectified background EMG was averaged in each trial over the 100 -ms period preceding the TMS artifact, and expressed as a percentage of the EMG activity measured during the MVC trial. The peak-to-peak amplitude of the TMS-generated MEPs was computed. Using the interquartile range (Tukey, 1977), four percent of the total number of trials were identified as outliers and were substituted with the mean. SICI and ICF were expressed as percentage inhibition and facilitation, by using the following formula for SICI: 100 - (conditioned MEP/test MEP $\times 100)$, and the following formula for ICF: (conditioned MEP/test MEP $\times 100$ ) 100. CoP velocity while standing on the rigid platform and on foam was used to describe the postural behavior of subjects. CoP velocity is a highly reliable measure (Demura et al., 2008) that has proven to discriminate well between age groups and test situations (Raymakers et al., 2005).

\section{STATISTICAL ANALYSES}

All variables were checked for normal distribution prior to analysis. Background EMG was logarithmically transformed because of a skewed distribution. We compared MT between young and old adults using an independent samples $t$-test. The main analysis was an Age (young, old) by Condition (rigid-EO, rigid-EC, foam-EO, foam-EC) two-way repeated measures ANOVA on test MEP amplitude, SICI, ICF, and background EMG. In case of a significant condition effect, results were subjected to a post hoc Tukey's test. Because CoP velocity was only measured in two conditions (rigid, foam), this outcome measure was analyzed in an Age (young, old) by Condition (rigid, foam) two-way repeated measures ANOVA. A Greenhouse-Geisser correction was applied when the assumption of sphericity was violated. Pearson correlation coefficients were computed to assess the association of background EMG with test MEP amplitude, SICI and ICF, and the association of test MEP amplitude with SICI, using the combined age group data. Furthermore, Pearson correlation coefficients were computed to examine the relationship between the changes in SICI and CoP velocity from rigid to foam conditions in the two age groups. IBM SPSS statistics 20 was used for statistical analysis. The alpha level was set at 0.05. Results are presented as mean $\pm \mathrm{SD}$ in the text and tables and mean $\pm \mathrm{SE}$ in Figures 1 and 3. Interaction effects are only reported when significant.

\section{RESULTS}

\section{CENTER OF PRESSURE}

There was a significant age $\times$ condition interaction effect for CoP velocity $\left(F_{1,19}=15.8 ; p=0.001\right.$; Figure 1$)$, with a greater increase in $\mathrm{CoP}$ velocity from the rigid to the foam condition in old adults (from 1.75 to $2.58 \mathrm{~cm} / \mathrm{s}, p<0.001$ ) as compared to young adults (from 1.54 to $1.85 \mathrm{~cm} / \mathrm{s}, p<0.001$ ).

\section{TMS MEASURES}

Across all subjects, MT was similar in young (49 $\pm 8 \%$, range $34-55 \%)$ and old adults $\left(50 \pm 12 \%\right.$, range $31-73 \%, t_{21}=-0.2$, $p=0.845)$. Therefore, the average stimulation intensities were similar in the two age groups. The effects of sensory condition on TMS responses from a representative young and old subject are illustrated in Figure 2. Group data show that test MEP amplitude was similar in young and old adults $\left(F_{1,20}=1.3\right.$, $p=0.261$; Figure 3A). However, there was a significant condition effect $\left(F_{2,39}=14.2, p<0.001\right)$. Post hoc tests revealed that the test MEP's during standing on foam were greater compared

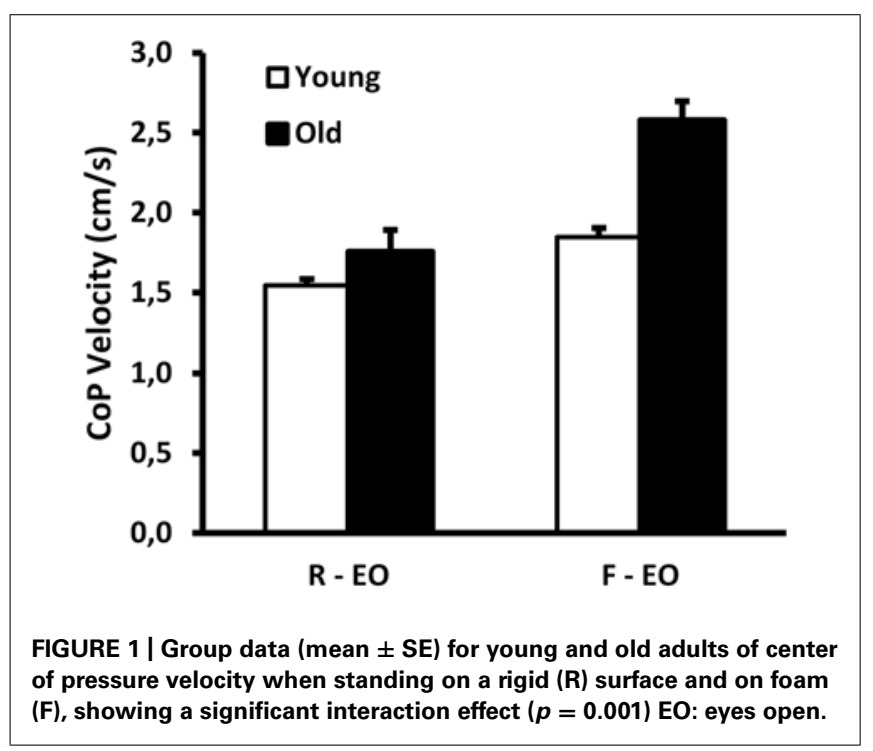




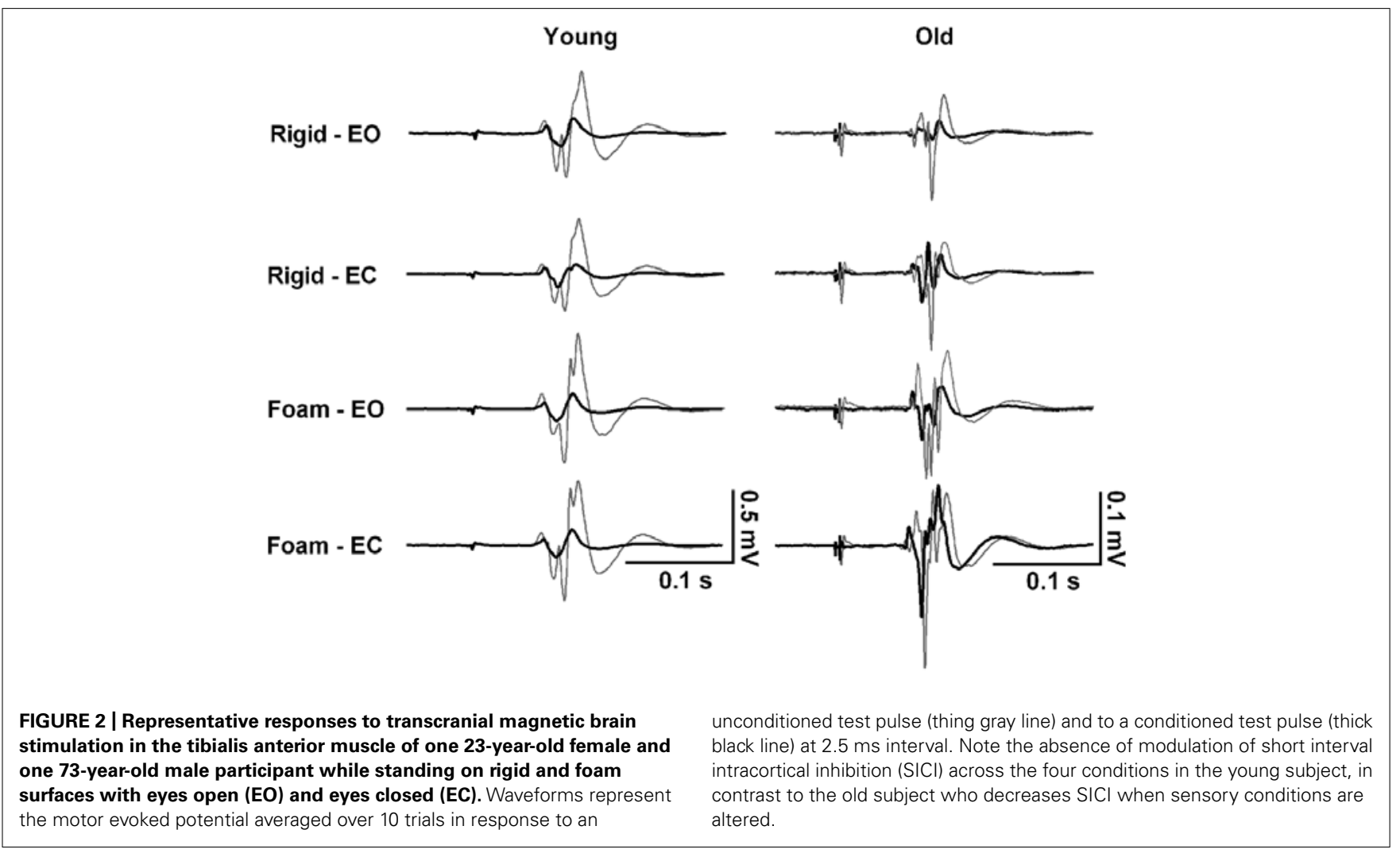

with those recorded while standing on a rigid surface $(p<0.01)$, and during foam-EC compared with foam-EO $(p<0.001)$. However, no difference related to vision conditions was observed when standing on a rigid surface $(p>0.05)$. The $2 \times 4$ ANOVA did not reveal significant modulation between conditions for SICI $\left(F_{3,60}=1.7, p=0.185\right)$, but SICI was greater in young adults $(67 \pm 23 \%)$ compared to old adults $(39 \pm 20 \%)$ regardless of standing condition $\left(F_{1,20}=16.5, p=0.001\right.$; Figure 3B). Furthermore, when pooled across vision conditions, the 2 (young, old) by 2 (rigid, foam) ANOVA revealed a significant age $\times$ condition interaction $\left(F_{1,20}=4.4, p=0.049\right)$, with a decrease in SICI from rigid to foam in old ( $31 \%$ change, $p<0.001)$ but not in young adults $(p>0.05)$. Age and conditions did not affect ICF $\left(F_{1,20}=0.4, p=0.527 ; F_{3,60}=1.2, p=0.325\right.$; Figure 3C).

\section{BACKGROUND EMG}

Figure 3D shows the group $\times$ condition interaction $\left(F_{2,34}=4.1\right.$; $p=0.031)$ for the log-transformed background EMG in the TA, with a greater increase in background EMG when altering the sensory conditions in old compared to young adults. Post hoc tests revealed that there was no effect of sensory condition on background EMG in young adults $(p>0.05)$. In old adults all comparisons between conditions were significant $(p<0.05)$, except for rigid-EO vs. rigidEC $(p>0.05)$. Moreover, bEMG did not differ between age groups during the rigid surface conditions $(p>0.05)$, but was higher in old adults during the foam surface conditions $(p<0.001)$.

\section{CORRELATIONS}

To determine whether differences in background EMG could have caused the condition (rigid vs. foam) and age (young vs. old) effects in TMS measures, we performed correlation analyses. The changes in background EMG between the rigid and the foam conditions did not correlate significantly with the changes in test MEP amplitude $(r=0.27, p=0.244)$, SICI $(r=-0.29, p=0.202)$ or $\operatorname{ICF}(r=-0.25, p=0.275)$. Furthermore, there was no significant correlation between the changes in test MEP amplitude and SICI $(r=-0.26, p=0.239)$ or ICF $(r=-0.17, p=0.444)$.

Figure 4 shows the relationship between changes in SICI and $\mathrm{CoP}$ velocity from the rigid to the foam condition in young and old adults. Within the old adults, subjects who reduced their SICI more when changing from standing on a rigid surface to standing on the foam, showed a greater increase in CoP velocity $(r=-0.65$, $p=0.043)$.

\section{DISCUSSION}

This is the first study that examines the effects of age on intracortical circuit excitability during postural tasks. The data confirmed the overriding hypothesis of an interaction between age and sensory condition in M1 excitability: SICI lessened when standing on foam compared with standing on a rigid surface in old but not in young adults. This reduction was associated with a greater decrease in stability when the support surface was altered. In contrast, ICF did not vary with age and sensory conditions. This study extends the literature reporting on the age-related reductions in cortical inhibition during manual tasks by demonstrating similar reductions in cortical inhibition during a postural task. 

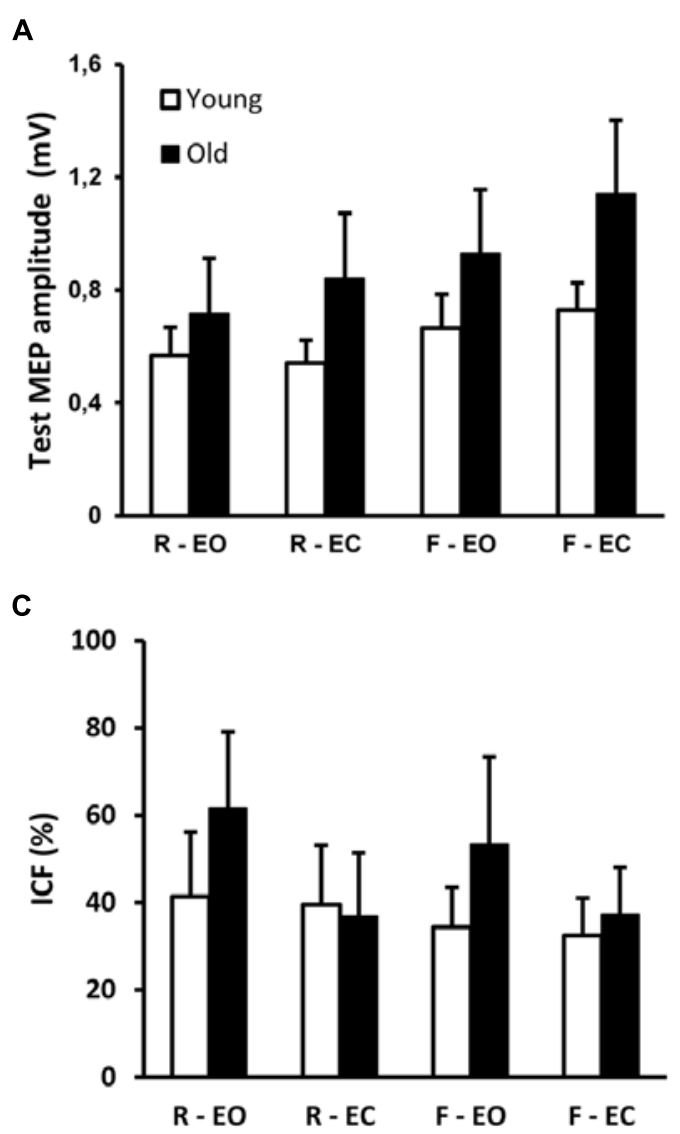

FIGURE 3 | Group data (mean \pm SE) for young and old adults of (A) amplitude test MEP (condition effect, $p<0.001$ ), (B) short interval intracortical inhibition ( $\mathrm{SICl}$; group effect, $p=0.001$; interaction effect, $p=0.049$ ), (C) intracortical facilitation (ICF),
B

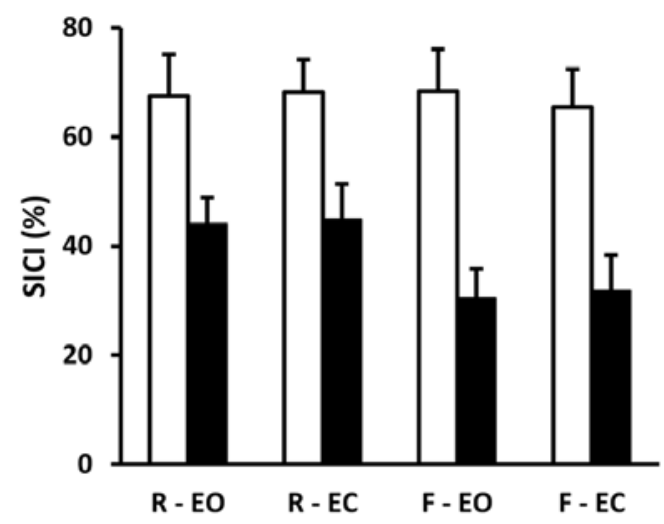

D

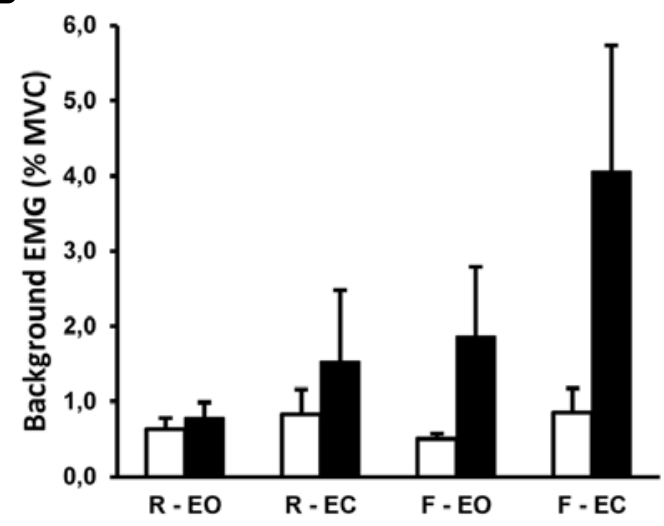

(D) background EMG (interaction effect, $\boldsymbol{p}=\mathbf{0 . 0 3 1}$ ). Conditions were standing on a rigid platform and on foam (rigid, foam), with eyes open and eyes closed (EO, EC). Greater values for $\mathrm{SICl}$ and ICF represent respectively more inhibition and facilitation.

\section{AGE-RELATED DECREASE IN SICI}

Previous studies reported inconsistent results concerning the association between age and SICI. The inconsistencies may in part be due to differences in the strength of corticomotoneuronal projections to the target muscle. For example, hand muscles compared with forearm muscles have more abundant monosynaptic projections (Palmer and Ashby, 1992), and SICI investigated in motor cortex associated with hand muscles has been reported to be reduced (Marneweck etal., 2011; Heise etal., 2013) or similar (Oliviero etal., 2006; Smith etal., 2011) in old compared with young adults. In contrast, studies examining wrist flexors and extensors found more SICI in old than in young adults (Kossev etal., 2002; McGinley et al., 2010). As the TA exhibits nearly similar density of corticospinal projections as hand muscles (Brouwer and Ashby, 1992; Petersen et al., 2003), results of the present study support the hypothesis of a specific reduction in SICI for muscles with rich corticospinal projections, suggesting that aging further strengthens the corticomotoneuronal pathway by diminishing intracortical inhibition. Nonetheless, this may also reflect a greater contribution of this pathway during upright standing, regardless of leg muscles (Baudry et al., 2014b).

\section{POSTURE-SPECIFIC REDUCTIONS IN SICI AFFECT PERFORMANCE}

In addition to the lower SICI in elderly adults, the present results show that elderly adults modulated SICI depending on the sensory conditions, and more specifically the surface of support. There are several studies showing changes in SICI related to motor control. For example, SICI is reduced during movement preparation (Heise et al., 2013), during the activation phase compared with the deactivation phase in cycling (Sidhu et al., 2013), and after periods of motor practice (Rosenkranz et al., 2007; Smyth et al., 2010). However, we found no difference in SICI between tasks in young adults, resulting in a significant age $\times$ condition interaction. It must be noted that this interaction was only significant when data was pooled across vision conditions, probably due to a relatively low number of participants. Also, this might indicate that the modulation only occurs when balance is truly challenged, as changing the surface usually has a bigger impact than removing vision (Baudry and Duchateau, 2012). The lack of modulation in SICI in young adults is in line with a previous study that reported similar SICI levels during voluntary and postural contraction of the soleus muscle, suggesting that SICI is not specifically modulated during upright standing in young adults (Soto et al., 2006). This 


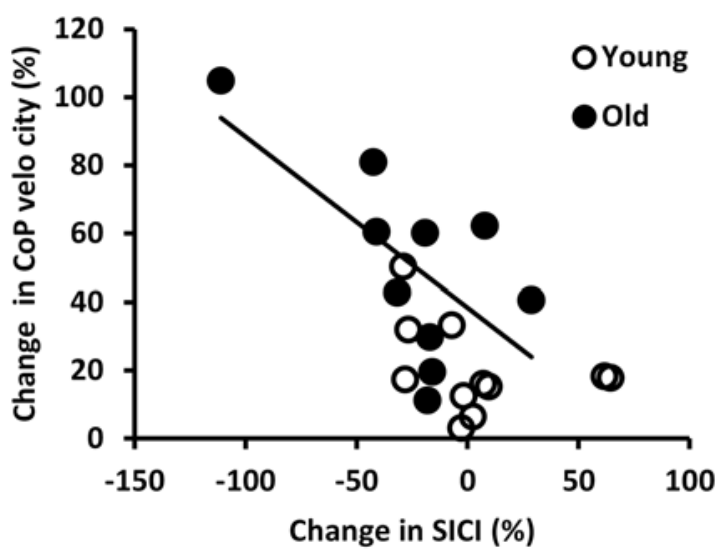

FIGURE 4 | Correlation between the percent changes in short interval intracortical inhibition (SICI) and center of pressure (CoP) velocity from the rigid to the foam condition in young and old adults. The regression line illustrates that in old subjects greater reductions in $\mathrm{SICl}$ were accompanied by greater increases in CoP velocity $(r=-0.648, p=0.043$ ).

may be because increasing M1 excitability would not improve muscle coordination needed to perform a difficult postural task. It may also be that the postural tasks were not challenging enough for the young adults to require changes in neural control, although young adults did increase CoP velocity when standing on foam.

Nonetheless, old adults exhibited a lower level of SICI when standing on foam compared to standing on a rigid surface. Within the group of old adults, reductions in SICI correlated with increases in CoP velocity. This is consistent with other studies reporting a correlation between low intracortical inhibition and poor motor performance in old adults (Marneweck et al., 2011; Fujiyama etal., 2012; Heise et al., 2013). Lower SICI was associated with poorer performance in the Purdue Pegboard test for manual dexterity, but not with performance on a pinch grip force control task (Marneweck et al., 2011). The correlation was driven by a number of (primarily old) subjects who exhibited atypically low inhibition, appearing as facilitation. There was also an association between the strength of intracortical inhibition measured by the contralateral silent period during an interlimb coordination task and the performance on this task (Fujiyama et al., 2012). At last, there was a link between the level of SICI at rest and single reaction time and two finger tapping speed (Heise et al., 2013). Although all three papers (Marneweck et al., 2011; Fujiyama et al., 2012; Heise et al., 2013) found an association between age-related disinhibition and reductions in motor performance, these studies did not quantify changes between tasks, instead reported inhibition only during one specific task (Marneweck et al., 2011; Fujiyama et al., 2012) or at rest (Heise et al., 2013). Therefore, the present study is the first to show that task-related modulation in SICI can be linked to behavior in old adults. One interpretation could be that the task-specific reduction in SICI was dysfunctional, negatively affecting postural control. As phasic activation of GABA-A receptors is thought to contribute to the regulation of synchronous motor neuronal activity (Farrant and Nusser, 2005), it is suggested that a general disinhibition could interfere with this process (Heise et al., 2013), and therefore induce difficulties when one is required to coordinate the fast contractions of different muscles during standing on foam.

However, we cannot exclude the possibility that the reduction in SICI in old adults was a compensatory mechanism. It can be argued that standing on foam is a relatively more difficult task for old than young subjects, and that the more subjects were challenged the more they were 'forced' to facilitate M1 activity. Therefore, neither the previous nor the current data can determine whether increased instability caused reduced SICI or the other way around.

\section{NO AGE-RELATED CHANGES IN ICF}

We did not find differences in ICF between young and old adults, which is consistent with two studies that examined ICF in the first dorsal interosseus muscle (Smith et al., 2009, 2011). However, it is inconsistent with two other studies that reported lower ICF in the wrist flexors and extensors of old compared with young adults (Kossev et al., 2002; McGinley et al., 2010). This is in line with the earlier proposed shift towards an age-related facilitation of the muscles with strong corticospinal projections. Given the decrease in SICI, the lack of change in ICF may indicate that modulation of cortical circuits with sensory conditions relies more on reducing strength of inhibitory inputs to increase the potential for more cortical contribution to control leg muscles rather than increasing cortical activity. This is in agreement with the lack of changes in test MEP observed in the present study and the absence of modulation in corticomotoneural excitability with altered proprioception (Baudry et al., 2014b). Such adjustments may be relevant to increase the cortical contribution to postural control without increasing cortical activity that may induce noisy neural signal.

Although many studies have used ICF to investigate cortical excitability (Ginanneschi et al., 2005; Soto et al., 2006; Smyth et al., 2010; Howatson et al., 2011), the neurophysiological origin of ICF is still under debate (Reis et al., 2008). As N-methyl-D-aspartate (NMDA) receptor antagonists and glutamate antagonists decrease ICF, it is often believed to reflect glutaminergic intracortical circuits (Liepert et al., 1997; Ziemann et al., 1998). However, there is some evidence that GABAergic inhibition can modulate ICF (Ziemann et al., 1996). Furthermore, spinal contributions cannot be excluded (Di Lazzaro et al., 2006). Therefore, conclusions regarding ICF should be used with caution, and short interval intracortical facilitation (SICF), an often overlooked measure, might actually be more valid for determining cortical facilitation (Di Lazzaro et al., 1999; Clark et al., 2011).

\section{THE INFLUENCE OF BACKGROUND EMG}

One limitation of this study is the difference in background EMG between conditions and groups. Since the level of muscle contraction could affect SICI, this might have influenced the results. However, there are several reasons why we think this is not the case. First, the contractions were of very low intensity, with an average of 0.9 and $4.1 \%$ of MVC in young and old adults during the most difficult condition. Second, the age-related decrease in SICI was also apparent in the rigid-EO condition, while the background EMG did not differ between groups in that condition. Third, the 
modulation between conditions in SICI did not correlate with the modulation of background EMG.

\section{FUTURE RECOMMENDATIONS AND CONCLUSIONS}

Future studies are needed to better understand the cause-effect relationship between SICI and postural control in aging. One aspect in these studies could be to test the hypothesis of an interaction between age and the strength of corticospinal projection to the target muscle on the amount of SICI and ICF by targeting several muscles. If this proves to be true, such data would address many inconsistencies reported in literature and provide a deeper insight into the age-related changes of the neuromotor system. Another aspect could be the association between behavioral and neurophysiological changes after balance training.

In conclusion, old compared with young adults exhibited an overall decreased level of SICI and reduced this even further when standing with altered proprioception. The reduction in SICI between tasks was associated with an increased velocity of the CoP. This suggests that motor cortical circuits control upright posture differently in old vs. young adults. Future experiments will clarify whether this difference in control mediates impaired postural control or serves as a compensatory mechanism to counteract postural instability.

\section{ACKNOWLEDGMENTS}

This work was supported by start-up funds from the University Medical Center Groningen. The authors thank Prof. E. Otten and Dr. H. G. van Keeken for their insightful comments, E. Smid for his technical assistance, and N. Kerver and L. Maas for helping with data collection.

\section{REFERENCES}

Baudry, S., and Duchateau, J. (2012). Age-related influence of vision and proprioception on ia presynaptic inhibition in soleus muscle during upright stance. J. Physiol. 590(Pt 21), 5541-5554. doi: 10.1113/jphysiol.2012. 228932

Baudry, S., Penzer, F., and Duchateau, J. (2014a). Input-output characteristics of soleus homonymous la afferents and corticospinal pathways during upright standing differ between young and elderly adults. Acta Physiol. (Oxf.) 210, 667-677. doi: 10.1111/apha.12233

Baudry, S., Penzer, F., and Duchateau, J. (2014b). Vision and proprioception do not influence the excitability of the corticomotoneuronal pathway during upright standing in young and elderly adults. Neuroscience 268, 247-254. doi: 10.1016/j.neuroscience.2014.03.026

Beck, S., Taube, W., Gruber, M., Amtage, F., Gollhofer, A., and Schubert, M. (2007). Task-specific changes in motor evoked potentials of lower limb muscles after different training interventions. Brain Res. 1179, 51-60. doi: 10.1016/j.brainres.2007.08.048

Beloozerova, I. N., Sirota, M. G., Swadlow, H. A., Orlovsky, G. N., Popova, L. B., and Deliagina, T. G. (2003). Activity of different classes of neurons of the motor cortex during postural corrections. J. Neurosci. 23, 7844-7853.

Boelens, C., Hekman, E. E., and Verkerke, G. J. (2013). Risk factors for falls of older citizens. Technol. Health Care 21, 521-533. doi: 10.3233/THC130748

Brouwer, B., and Ashby, P. (1992). Corticospinal projections to lower limb motoneurons in man. Exp. Brain Res. 89, 649-654. doi: 10.1007/ BF00229889

Cacchio, A., Cimini, N., Alosi, P., Santilli, V., and Marrelli, A. (2009). Reliability of transcranial magnetic stimulation-related measurements of tibialis anterior muscle in healthy subjects. Clin. Neurophysiol. 120, 414-419. doi: 10.1016/j.clinph.2008.11.019
Calautti, C., Serrati, C., and Baron, J. C. (2001). Effects of age on brain activation during auditory-cued thumb-to-index opposition: a positron emission tomography study. Stroke 32, 139-146. doi: 10.1161/01.STR. 32.1.139

Clark, J., Loftus, A., and Hammond, G. (2011). Age-related changes in shortinterval intracortical facilitation and dexterity. Neuroreport 22, 499-503. doi: 10.1097/WNR.0b013e3283487480

Demura, S., Kitabayashi, T., and Aoki, H. (2008). Body-sway characteristics during a static upright posture in the elderly. Geriatr. Gerontol. Int. 8, 188-197. doi: 10.1111/j.1447-0594.2008.00469.x

Di Lazzaro, V., Pilato, F., Oliviero, A., Dileone, M., Saturno, E., Mazzone, P., et al. (2006). Origin of facilitation of motor-evoked potentials after paired magnetic stimulation: direct recording of epidural activity in conscious humans. $J$. Neurophysiol. 96, 1765-1771. doi: 10.1152/jn.00360.2006

Di Lazzaro, V., Rothwell, J. C., Oliviero, A., Profice, P., Insola, A., Mazzone, P., et al. (1999). Intracortical origin of the short latency facilitation produced by pairs of threshold magnetic stimuli applied to human motor cortex. Exp. Brain Res. 129, 494-499. doi: 10.1007/s002210050919

Farrant, M., and Nusser, Z. (2005). Variations on an inhibitory theme: phasic and tonic activation of GABA(A) receptors. Nat. Rev. Neurosci. 6, 215-229. doi: $10.1038 / \mathrm{nrn} 1625$

Fujiyama, H., Hinder, M. R., Schmidt, M. W., Garry, M. I., and Summers, J. J. (2012). Age-related differences in corticospinal excitability and inhibition during coordination of upper and lower limbs. Neurobiol. Aging 33, 1484.e1-1484.e14. doi: 10.1016/j.neurobiolaging.2011.12.019

Ginanneschi, F., Del Santo, F., Dominici, F., Gelli, F., Mazzocchio, R., and Rossi, A. (2005). Changes in corticomotor excitability of hand muscles in relation to static shoulder positions. Exp. Brain Res. 161, 374-382. doi: 10.1007/s00221-0042084-X

Hebbal, G. V., and Mysorekar, V. R. (2006). Evaluation of some tasks used for specifying handedness and footedness. Percept. Mot. Skills 102, 163-164. doi: 10.2466/pms.102.1.163-164

Heise, K. F., Zimerman, M., Hoppe, J., Gerloff, C., Wegscheider, K., and Hummel, F. C. (2013). The aging motor system as a model for plastic changes of GABAmediated intracortical inhibition and their behavioral relevance. J. Neurosci. 33, 9039-9049. doi: 10.1523/JNEUROSCI.4094-12.2013

Hortobagyi, T., del Olmo, M. F., and Rothwell, J. C. (2006). Age reduces cortical reciprocal inhibition in humans. Exp. Brain Res. 171, 322-329. doi: 10.1007/s00221-005-0274-9

Howatson, G., Taylor, M. B., Rider, P., Motawar, B. R., McNally, M. P., Solnik, S., et al. (2011). Ipsilateral motor cortical responses to TMS during lengthening and shortening of the contralateral wrist flexors. Eur. J. Neurosci. 33, 978-990. doi: 10.1111/j.1460-9568.2010.07567.x

Katz, R., Meunier, S., and Pierrot-Deseilligny, E. (1988). Changes in presynaptic inhibition of ia fibres in man while standing. Brain 111, 417-437. doi: 10.1093/brain/111.2.417

Koceja, D. M., Markus, C. A., and Trimble, M. H. (1995). Postural modulation of the soleus $\mathrm{H}$ reflex in young and old subjects. Electroencephalogr. Clin. Neurophysiol. 97, 387-393. doi: 10.1016/0924-980X(95) 00163-F

Kossev, A. R., Schrader, C., Dauper, J., Dengler, R., and Rollnik, J. D. (2002). Increased intracortical inhibition in middle-aged humans; a study using pairedpulse transcranial magnetic stimulation. Neurosci. Lett. 333, 83-86. doi: 10.1016/S0304-3940(02)00986-2

Liepert, J., Schwenkreis, P., Tegenthoff, M., and Malin, J. P. (1997). The glutamate antagonist riluzole suppresses intracortical facilitation. J. Neural Transm. 104, 1207-1214. doi: 10.1007/BF01294721

Marneweck, M., Loftus, A., and Hammond, G. (2011). Short-interval intracortical inhibition and manual dexterity in healthy aging. Neurosci. Res. 70, 408-414. doi: 10.1016/j.neures.2011.04.004

Mattay, V. S., Fera, F., Tessitore, A., Hariri, A. R., Das, S., Callicott, J. H., etal. (2002). Neurophysiological correlates of age-related changes in human motor function. Neurology 58, 630-635. doi: 10.1212/WNL. 58.4.630

McGinley, M., Hoffman, R. L., Russ, D. W., Thomas, J. S., and Clark, B. C. (2010). Older adults exhibit more intracortical inhibition and less intracortical facilitation than young adults. Exp. Gerontol. 45, 671-678. doi: 10.1016/j.exger.2010. 04.005 
Nardone, A., Siliotto, R., Grasso, M., and Schieppati, M. (1995). Influence of aging on leg muscle reflex responses to stance perturbation. Arch. Phys. Med. Rehabil. 76, 158-165. doi: 10.1016/S0003-9993(95) 80025-5

Oliviero, A., Profice, P., Tonali, P. A., Pilato, F., Saturno, E., Dileone, M., et al. (2006). Effects of aging on motor cortex excitability. Neurosci. Res. 55, 74-77. doi: 10.1016/j.neures.2006.02.002

Palmer, E., and Ashby, P. (1992). Corticospinal projections to upper limb motoneurones in humans. J. Physiol. 448, 397-412.

Papegaaij, S., Taube, W., Baudry, S., Otten, E., and Hortobagyi, T. (2014). Aging causes a reorganization of cortical and spinal control of posture. Front. Aging Neurosci. 6:28. doi: 10.3389/fnagi.2014.00028

Petersen, N. T., Pyndt, H. S., and Nielsen, J. B. (2003). Investigating human motor control by transcranial magnetic stimulation. Exp. Brain Res. 152, 1-16. doi 10.1007/s00221-003-1537-y

Proske, U., and Gandevia, S. C. (2012). The proprioceptive senses: their roles in signaling body shape, body position and movement, and muscle force. Physiol. Rev. 92, 1651-1697. doi: 10.1152/physrev. 00048.2011

Raymakers, J. A., Samson, M. M., and Verhaar, H. J. (2005). The assessment of body sway and the choice of the stability parameter(s). Gait Posture 21, 48-58. doi: 10.1016/j.gaitpost.2003.11.006

Reis, J., Swayne, O. B., Vandermeeren, Y., Camus, M., Dimyan, M. A., HarrisLove, M., et al. (2008). Contribution of transcranial magnetic stimulation to the understanding of cortical mechanisms involved in motor control. J. Physiol. 586, 325-351. doi: 10.1113/jphysiol.2007.144824

Rosenkranz, K., Kacar, A., and Rothwell, J. C. (2007). Differential modulation of motor cortical plasticity and excitability in early and late phases of human motor learning. J. Neurosci. 27, 12058-12066. doi: 10.1523/JNEUROSCI.266307.2007

Roshan, L., Paradiso, G. O., and Chen, R. (2003). Two phases of short-interval intracortical inhibition. Exp. Brain Res. 151, 330-337. doi: 10.1007/s00221-0031502-1509

Schubert, M., Beck, S., Taube, W., Amtage, F., Faist, M., and Gruber, M. (2008) Balance training and ballistic strength training are associated with task-specific corticospinal adaptations. Eur. J. Neurosci. 27, 2007-2018. doi: 10.1111/j.14609568.2008.06186.x

Sidhu, S. K., Cresswell, A. G., and Carroll, T. J. (2013). Short-interval intracortical inhibition in knee extensors during locomotor cycling. Acta Physiol. (Oxf.) 207, 194-201. doi: 10.1111/apha.12004

Smith, A. E., Ridding, M. C., Higgins, R. D., Wittert, G. A., and Pitcher, J. B. (2009). Age-related changes in short-latency motor cortex inhibition. Exp. Brain Res. 198, 489-500. doi: 10.1007/s00221-0091945-8

Smith, A. E., Ridding, M. C., Higgins, R. D., Wittert, G. A., and Pitcher, J. B. (2011). Cutaneous afferent input does not modulate motor intracortical inhibition in ageing men. Eur. J. Neurosci. 34, 1461-1469. doi: 10.1111/j.1460-9568.2011. 07869.x

Smyth, C., Summers, J. J., and Garry, M. I. (2010). Differences in motor learning success are associated with differences in M1 excitability. Hum. Mov. Sci. 29, 618-630. doi: 10.1016/j.humov.2010.02.006
Soto, O., Valls-Sole, J., Shanahan, P., and Rothwell, J. (2006). Reduction of intracortical inhibition in soleus muscle during postural activity. J. Neurophysiol. 96 1711-1717. doi: 10.1152/jn.00133.2006

Talelli, P., Waddingham, W., Ewas, A., Rothwell, J. C., and Ward, N. S. (2008). The effect of age on task-related modulation of interhemispheric balance. Exp. Brain Res. 186, 59-66. doi: 10.1007/s00221-007-1205-1208

Taube, W., Gruber, M., Beck, S., Faist, M., Gollhofer, A., and Schubert, M. (2007). Cortical and spinal adaptations induced by balance training: correlation between stance stability and corticospinal activation. Acta Physiol.(Oxf). 189, 347-358. doi: 10.1111/j.1365-201X.2007.01665.x

Taube, W., Gruber, M., and Gollhofer, A. (2008). Spinal and supraspinal adaptations associated with balance training and their functional relevance. Acta Physiol.(Oxf). 193, 101-116. doi: 10.1111/j.1748-1716.2008. 01850.x

Taube, W., Schubert, M., Gruber, M., Beck, S., Faist, M., and Gollhofer, A. (2006). Direct corticospinal pathways contribute to neuromuscular control of perturbed stance. J. Appl. Physiol. 101, 420-429. doi: 10.1152/japplphysiol. 01447.2005

Taubert, M., Draganski, B., Anwander, A., Muller, K., Horstmann, A., Villringer, A., et al. (2010). Dynamic properties of human brain structure: learning-related changes in cortical areas and associated fiber connections. J. Neurosci. 30, 1167011677. doi: 10.1523/JNEUROSCI.2567-10.2010

Tokuno, C. D., Taube, W., and Cresswell, A. G. (2009). An enhanced level of motor cortical excitability during the control of human standing. Acta Physiol. (Oxf). 195, 385-395. doi: 10.1111/j.1748-1716.2008.01898.x

Tukey, J. W. (1977). Exploratory Data Analysis. Reading, MA: Addison-Wesley.

Ward, N. S., and Frackowiak, R. S. (2003). Age-related changes in the neural correlates of motor performance. Brain 126, 873-888. doi: 10.1093/brain/awg071 Ziemann, U., Chen, R., Cohen, L. G., and Hallett, M. (1998). Dextromethorphan decreases the excitability of the human motor cortex. Neurology 51, 1320-1324. doi: 10.1212/WNL.51.5.1320

Ziemann, U., Lonnecker, S., Steinhoff, B. J., and Paulus, W. (1996). The effect of lorazepam on the motor cortical excitability in man. Exp. Brain Res. 109, 127-135. doi: $10.1007 / \mathrm{BF} 00228633$

Conflict of Interest Statement: The authors declare that the research was conducted in the absence of any commercial or financial relationships that could be construed as a potential conflict of interest.

Received: 17 April 2014; paper pending published: 07 May 2014; accepted: 28 May 2014; published online: 12 June 2014.

Citation: Papegaaij S, Taube W, Hogenhout M, Baudry S and Hortobágyi T (2014) Agerelated decrease in motor cortical inhibition during standing under different sensory conditions. Front. Aging Neurosci. 6:126. doi: 10.3389/fnagi.2014.00126

This article was submitted to the journal Frontiers in Aging Neuroscience.

Copyright (c) 2014 Papegaaij, Taube, Hogenhout, Baudry and Hortobágyi. This is an open-access article distributed under the terms of the Creative Commons Attribution License (CC BY). The use, distribution or reproduction in other forums is permitted, provided the original author(s) or licensor are credited and that the original publication in this journal is cited, in accordance with accepted academic practice. No use, distribution or reproduction is permitted which does not comply with these terms. 